\title{
GLOBAL EXPONENTIAL STABILITY CRITERIA FOR SWITCHED NEUTRAL SYSTEMS WITH INTERVAL TIME-VARYING DELAY
}

Ker-Wei Yu

Department of Marine Engineering, National Kaohsiung Marine University, Kaohsiung, Taiwan, R.O.C., kwyu@mail.nkmu.edu.tw

Follow this and additional works at: https://jmstt.ntou.edu.tw/journal

Part of the Engineering Commons

\section{Recommended Citation}

Yu, Ker-Wei (2010) "GLOBAL EXPONENTIAL STABILITY CRITERIA FOR SWITCHED NEUTRAL SYSTEMS WITH INTERVAL TIME-VARYING DELAY," Journal of Marine Science and Technology. Vol. 18: Iss. 2, Article 17.

DOI: $10.51400 / 2709-6998.2330$

Available at: https://jmstt.ntou.edu.tw/journal/vol18/iss2/17

This Research Article is brought to you for free and open access by Journal of Marine Science and Technology. It has been accepted for inclusion in Journal of Marine Science and Technology by an authorized editor of Journal of Marine Science and Technology. 


\section{GLOBAL EXPONENTIAL STABILITY CRITERIA FOR SWITCHED NEUTRAL SYSTEMS WITH INTERVAL TIME-VARYING DELAY}

Acknowledgements

The research reported here was supported by the National Science Council of Taiwan, R.O.C. under grant no. NSC 96- 2221-E-022-011-MY2. 


\title{
GLOBAL EXPONENTIAL STABILITY CRITERIA FOR SWITCHED NEUTRAL SYSTEMS WITH INTERVAL TIME-VARYING DELAY
}

\author{
Ker-Wei Yu*
}

Key words: switched systems, neutral systems, global exponential stability, interval time-varying state delay, Razumikhinlike approach.

\begin{abstract}
The delay-dependent and delay-independent conditions are proposed to guarantee the global exponential stability for uncertain switched neutral system with interval time-varying state delay. New additional nonnegative inequalities are introduced to improve the conservativeness of system. Razumikhinlike approach is used to prove the exponential stability for system. Structured and unstructured uncertainties are investigated in this paper. The solving schemes based on Linear Matrix Inequality (LMI) approach along with the selective examples are presented to demonstrate the improvements achieved.
\end{abstract}

\section{INTRODUCTION}

A switched system is a class of hybrid systems which consists of several subsystems and exhibits the switching feature between multi-models, which is usually used to approximate many practical nonlinear systems [10]. It is well known that the existence of time delay in a system may cause instability or bad system performance in feedback control systems. Since time-delay phenomenon may encounter in many practical systems, such as aircraft stabilization, chemical engineering systems, inferred grinding model, neural network, nuclear reactor, population dynamic model, rolling mill, ship stabilization, and systems with lossless transmission lines [2, 3, 5]. Hence stability analysis for switched time-delay systems has been investigated in recent years [4-9, 11, 13].

It is interesting to note that for each stable subsystem cannot imply that the overall system is also stable [7]. Hence we will consider the global exponential stability problem for

Paper submitted 04/07/09; revised 06/28/09; accepted 07/03/09. Author for correspondence: Ker-Wei, Yu (e-mail: kwyu@mail.nkmu.edu.tw).

*Department of Marine Engineering, National Kaohsiung Marine University, Kaohsiung, Taiwan, R.O.C. switched neutral systems with interval time-varying state delay and two classes of perturbations under arbitrary switched signal. Based on Razumikhin-like [12] and LMI approaches [1], delay-dependent and delay-independent results are provided. The LMI approach [1] is an efficient and powerful tool in solving some control problems; such as $H_{\infty}$ control, stability analysis, guaranteed cost control, state feedback control, static output feedback control, and observer-based control. Hence LMI approach will be used to guarantee the stability problem of systems. Some numerical examples are provided to demonstrate the main proposed results.

The notation used throughout this paper is as follows. For a matrix $A$, we denote the transpose by $A^{T}$, spectral norm by $\|A\|$, minimal (maximal) eigenvalue by $\lambda_{\text {min }}(A)\left(\lambda_{\max }(A)\right)$, symmetric positive (negative) definite by $A>0(A>0) . A \leq B$ means that matrix $B-A$ is symmetric positive semi-definite. For a vector $x$, we denote the Euclidean norm by $\|x\|$. For the state $x_{t}$ of system, we define $x_{t}(\theta):=x(t+\theta), \forall \theta \in[-H, 0]$ and denote its norm by $\left\|x_{t}\right\|_{s}=\sup _{-H \leq \theta \leq 0} \sqrt{\|x(t+\theta)\|^{2}+\|\dot{x}(t+\theta)\|^{2}}$. $I$ denotes the identity matrix.

\section{PROBLEM FORMULATION AND MAIN RESULTS}

Consider the following uncertain switched neutral system with interval time-varying state delay:

$$
\begin{gathered}
\dot{x}(t)-D \dot{x}(t-\tau)=\left[A_{0 \sigma}+\Delta A_{0 \sigma}(t)\right] x(t) \\
+\left[A_{1 \sigma}+\Delta A_{1 \sigma}(t)\right] x(t-h(t)), t \geq 0, \\
x(t)=\phi(t), t \in[-H, 0],
\end{gathered}
$$

where $x \in \mathfrak{R}^{n}$. Switching signal $\sigma$ may depend on $t$ or $x$ and takes its values in the finite set $\{i=1,2, \cdots, N\}$. Interval time-varying delay $h(t)$ satisfies $0 \leq h_{m}<h(t) \leq h_{M}, \dot{h}(t) \leq h_{D}$. Constant delay $\tau>0$ and $H=\max \left\{h_{M}, \tau\right\}$. Matrices $D, A_{0 i}$, and $A_{1 i} \in \Re^{n \times n}, i=1,2, \cdots, N$, are constant, and the initial vector $\phi \in C_{1}$, where $C_{1}$ is the set of differentiable functions from $[-H, 0]$ to $\mathfrak{R}^{n}$. 
In this paper, we will consider the following two types of perturbations on system:

(A1) Structured perturbations: $\Delta A_{0 i}(t)$ and $\Delta A_{1 i}(t)$ satisfy the following conditions

$$
\begin{gathered}
{\left[\Delta A_{0 i}(t) \quad \Delta A_{1 i}(t)\right]=M_{i} F_{i}(t)\left[\begin{array}{ll}
N_{0 i} & N_{1 i}
\end{array}\right],} \\
\forall i \in\{1,2, \cdots, N\}, t \geq 0,
\end{gathered}
$$

where $M_{i}, N_{0 i}$, and $N_{1 i}, i=1,2, \cdots, N$, are some given constant matrices with appropriate dimensions, and $F_{i}(t)$, $i=1,2, \cdots, N$, are unknown matrices representing the parameter perturbation which satisfy

$$
F_{i}^{T}(t) F_{i}(t) \leq I, \forall i \in\{1,2, \cdots, N\}, t \geq 0 .
$$

(A2) Unstructured perturbations: $\Delta A_{0 i}(t)$ and $\Delta A_{1 i}(t)$ satisfy the following conditions

$$
\left\|\Delta A_{0 i}(t)\right\| \leq \sigma_{0 i} \text { and }\left\|\Delta A_{1 i}(t)\right\| \leq \sigma_{1 i},
$$

where $\sigma_{0 i}$ and $\sigma_{1 i}, i=1,2, \cdots, N$, are some given nonnegative constants.

Define the functions $\lambda_{i}(t), i=1,2, \cdots, N$, as follows:

$$
\lambda_{i}(t)=\left\{\begin{array}{cc}
1, & \sigma=i, \\
0, & \text { otherwise }
\end{array} \quad i=1,2, \cdots, N\right.
$$

We can rewrite the switched system (1) to the following form:

$$
\begin{aligned}
\dot{x}(t)-D \dot{x}(t-\tau)= & \sum_{i=1}^{N} \lambda_{i}(t)\left\{\left[A_{0 i}+\Delta A_{0 i}(t)\right] x(t)\right. \\
& \left.+\left[A_{1 i}+\Delta A_{1 i}(t)\right] x(t-h(t))\right\}, t \geq 0, \\
& x(t)=\phi(t), \quad t \in[-H, 0],
\end{aligned}
$$

where $\lambda_{i}(t)$ is defined in (4) and $\sum_{i=1}^{N} \lambda_{i}(t)=1, \lambda_{i}^{2}(t)=\lambda_{i}(t)$, and $\lambda_{i}(t) \cdot \lambda_{j}(t)=0, i \neq j, \quad \forall t \geq 0$.

The following lemma will be used in the proof of our main results.

Lemma 1: [12] Let $U, V, W$ and $M$ be real matrices of appropriate dimensions with $M$ satisfying $M=M^{T}$, then

$$
M+U V W+W^{T} V^{T} U^{T}<0, \text { for all } V^{T} V \leq I,
$$

if and only if there exists a scalar $\varepsilon>0$ such that

$$
\begin{aligned}
M & +\varepsilon^{-1} \cdot U U^{T}+\varepsilon \cdot W^{T} W \\
& =M+\varepsilon^{-1} \cdot U U^{T}+\varepsilon^{-1} \cdot(\varepsilon W)^{T}(\varepsilon W)<0 .
\end{aligned}
$$

Definition 1. The system (1) with (A1) or (A2) is said to be the globally exponentially stable with convergence rate $\alpha>0$, if there are two positive constants $\alpha$ and $\Psi$ such that

$$
\|x(t)\| \leq \Psi \cdot e^{-\alpha t}, \quad t \geq 0
$$

Now we present a delay-dependent condition for stability of system (1) with (A1).

Theorem 1. System (1) with (A1) and $h_{D}<1$ (resp., $h_{D} \geq 1$ or unknown) is globally exponentially stable with convergence rate $0<\alpha<-(\ln \|D\|) / \tau$, if $\|D\|<1$ and there exist some $n \times n$ matrices $P, Q_{1}, Q_{2}, R_{1}, R_{2}, R_{3}, R_{4}, S, R_{22}, S_{22}, T_{22}>0$, $R_{11}, S_{11}, T_{11}>0 \in \mathfrak{R}^{7 n \times 7 n}$ (resp., $Q_{2}=0$ ), some matrices $U_{1 i}$, $U_{2 i}, U_{3 i}, U_{4 i}, U_{5 i}, U_{6 i}, U_{7 i} \in \Re^{n \times n}, R_{12}, S_{12}, T_{12} \in \Re^{7 n \times n}$, and some positive constants $\varepsilon_{i}, i=1,2, \cdots, N$, such that the following LMI conditions hold for all $i=1,2, \cdots, N$

$$
\begin{aligned}
& e^{-2 \alpha h_{m}} \cdot R_{1}-R_{22}>0, e^{-2 \alpha h_{M}} \cdot R_{2}-S_{22}>0, \\
& e^{-2 \alpha h_{M}} \cdot R_{2}-T_{22}>0 \text {, } \\
& {\left[\begin{array}{cc}
R_{11} & R_{12} \\
* & R_{22}
\end{array}\right]>0,\left[\begin{array}{cc}
S_{11} & S_{12} \\
* & S_{22}
\end{array}\right]>0,\left[\begin{array}{cc}
T_{11} & T_{12} \\
* & T_{22}
\end{array}\right]>0,} \\
& \hat{\Sigma}_{i}=\left[\begin{array}{ccccccccc}
\Sigma_{11 i} & \Sigma_{12 i} & \Sigma_{13 i} & \Sigma_{14 i} & \Sigma_{15 i} & \Sigma_{16 i} & \Sigma_{17 i} & \Sigma_{18 i} & \Sigma_{19 i} \\
* & \Sigma_{22 i} & \Sigma_{23 i} & 0 & 0 & 0 & 0 & \Sigma_{28 i} & 0 \\
* & * & \Sigma_{33 i} & \Sigma_{34 i} & \Sigma_{35 i} & \Sigma_{36 i} & \Sigma_{37 i} & \Sigma_{38 i} & \Sigma_{39 i} \\
* & * & * & \Sigma_{44 i} & \Sigma_{45 i} & \Sigma_{46 i} & 0 & \Sigma_{48 i} & 0 \\
* & * & * & * & \Sigma_{55 i} & \Sigma_{56 i} & \Sigma_{57 i} & \Sigma_{58 i} & 0 \\
* & * & * & * & * & \Sigma_{66 i} & \Sigma_{67 i} & \Sigma_{68 i} & 0 \\
* & * & * & * & * & * & \Sigma_{77 i} & \Sigma_{78 i} & 0 \\
* & * & * & * & * & * & * & \Sigma_{88 i} & 0 \\
* & * & * & * & * & * & * & * & \Sigma_{99 i}
\end{array}\right] \\
& +\left[\begin{array}{ccc}
\Phi_{11} & 0 & 0 \\
0 & 0 & 0 \\
0 & 0 & 0
\end{array}\right]<0
\end{aligned}
$$

where * represents the symmetric form in the matrix and $\Sigma_{11 i}=2 \alpha \cdot P+P A_{0 i}+A_{0 i}^{T} P+Q_{1}+Q_{2}+U_{2 i}^{T} A_{0 i}+A_{0 i}^{T} U_{2 i}+S+R_{4}$, 


$$
\begin{aligned}
& \Sigma_{12 i}=-2 \alpha \cdot P D-A_{0 i}^{T} P D, \\
& \Sigma_{13 i}=P A_{1 i}+U_{2 i}^{T} A_{1 i}+A_{0 i}^{T} U_{3 i}, \Sigma_{14 i}=A_{0 i}^{T} U_{6 i}, \\
& \Sigma_{15 i}=A_{0 i}^{T} U_{1 i}^{T}-U_{2 i}^{T}+A_{0 i}^{T} U_{4 i} \\
& \Sigma_{16 i}=-A_{0 i}^{T} U_{1 i}^{T} D+U_{2 i}^{T} D+A_{0 i}^{T} U_{5 i}, \Sigma_{17 i}=A_{0 i}^{T} U_{7 i}, \\
& \Sigma_{18 i}=P M_{i}+U_{2 i}^{T} M_{i}, \Sigma_{19 i}=\varepsilon_{i} \cdot N_{0 i}^{T}, \\
& \Sigma_{22 i}=2 \alpha \cdot D^{T} P D-e^{-2 \alpha \tau} \cdot S, \Sigma_{23 i}=-D^{T} P A_{1 i}, \\
& \Sigma_{28 i}=-D^{T} P M_{i}, \\
& \Sigma_{33 i}=-e^{-2 \alpha h_{M}} \cdot\left(1-h_{D}\right) \cdot Q_{2}+U_{3 i}^{T} A_{1 i}+A_{1 i}^{T} U_{3 i}, \Sigma_{34 i}=A_{1 i}^{T} U_{6 i}, \\
& \Sigma_{35 i}=A_{1 i}^{T} U_{1 i}^{T}-U_{3 i}^{T}+A_{1 i}^{T} U_{4 i}, \\
& \Sigma_{36 i}=-A_{1 i}^{T} U_{1 i}^{T} D+U_{3 i}^{T} D+A_{1 i}^{T} U_{5 i}, \Sigma_{37 i}=A_{1 i}^{T} U_{7 i}, \Sigma_{38 i}=U_{3 i}^{T} M_{i}, \\
& \Sigma_{39 i}=\varepsilon_{i} \cdot N_{1 i}^{T}, \Sigma_{44 i}=-e^{-2 \alpha h_{m}} \cdot Q_{1}+e^{-2 \alpha h_{m}} R_{3}, \\
& \Sigma_{45 i}=-U_{6 i}^{T}, \Sigma_{46 i}=U_{6 i}^{T} D, \Sigma_{48 i}=U_{6 i}^{T} M_{i}, \\
& \Sigma_{55 i}=h_{m} \cdot R_{1}+\left(h_{M}-h_{m}\right) \cdot R_{2}-U_{1 i}-U_{1 i}^{T}-U_{4 i}-U_{4 i}^{T}, \\
& \Sigma_{56 i}=\left(U_{1 i}+U_{1 i}^{T}\right) D+U_{4 i}^{T} D-U_{5 i}, \Sigma_{57 i}=-U_{7 i}, \\
& \Sigma_{58 i}=U_{1 i} M_{i}+U_{4 i}^{T} M_{i}, \\
& \Sigma_{66 i}=-D^{T}\left(U_{1 i}+U_{1 i}^{T}\right) D+U_{5 i}^{T} D+D^{T} U_{5 i}, \Sigma_{67 i}=D^{T} U_{7 i}, \\
& \Sigma_{68 i}=-D^{T} U_{1 i} M_{i}+U_{5 i}^{T} M_{i}, \Sigma_{77 i}=-e^{-2 \alpha h_{M}}\left(R_{3}+R_{4}\right), \\
& \Sigma_{78 i}=U_{7 i}^{T} M_{i}, \Sigma_{88 i}=\Sigma_{99 i}=-\varepsilon_{i} \cdot I \\
& \Phi_{11}=h_{m} \cdot R_{11}+R_{12} \Lambda_{1}+\Lambda_{1}^{T} R_{12}^{T}+\left(h_{M}-h_{m}\right) \cdot\left(T_{11}+S_{11}\right) \\
& +S_{12} \Lambda_{2}+\Lambda_{2}^{T} S_{12}^{T}+T_{12} \Lambda_{3}+\Lambda_{3}^{T} T_{12}^{T}, \\
& \Lambda_{1}=\left[\begin{array}{lllllll}
I & 0 & 0 & -I & 0 & 0 & 0
\end{array}\right]_{n \times 7 n}, \\
& \Lambda_{2}=\left[\begin{array}{lllllll}
0 & 0 & I & 0 & 0 & 0 & -I
\end{array}\right]_{n \times 7 n}, \\
& \Lambda_{3}=\left[\begin{array}{lllllll}
0 & 0 & -I & I & 0 & 0 & 0_{n \times 7 n} .
\end{array} .\right.
\end{aligned}
$$

Proof. Define the Lyapunov functional

$$
\begin{aligned}
V\left(x_{t}\right)= & e^{2 \alpha t} \cdot(x(t)-D x(t-\tau))^{T} P(x(t)-D x(t-\tau)) \\
& +\int_{t-h_{m}}^{t} e^{2 \alpha s} \cdot x^{T}(s) Q_{1} x(s) d s \\
& +\int_{t-h(t)}^{t} e^{2 \alpha s} \cdot x^{T}(s) Q_{2} x(s) d s \\
& +\int_{t-\tau}^{t} e^{2 \alpha s} \cdot x^{T}(s) S x(s) d s \\
& +\int_{t-h_{m}}^{t} e^{2 \alpha s} \cdot\left(s-\left(t-h_{m}\right)\right) \dot{x}^{T}(s) R_{1} \dot{x}(s) d s \\
& +\int_{t-h_{M}}^{t-h_{m}} e^{2 \alpha s} \cdot\left(s-\left(t-h_{M}\right)\right) \dot{x}^{T}(s) R_{2} \dot{x}(s) d s \\
& +\left(h_{M}-h_{m}\right) \cdot \int_{t-h_{m}}^{t} e^{2 \alpha s} \cdot \dot{x}^{T}(s) R_{2} \dot{x}(s) d s \\
& +\int_{t-h_{M}}^{t-h_{m}} e^{2 \alpha s} \cdot x^{T}(s) R_{3} x(s) d s \\
& +\int_{t-h_{M}}^{t} e^{2 \alpha s} \cdot x^{T}(s) R_{4} x(s) d s
\end{aligned}
$$

where $P, Q_{1}, Q_{2}, R_{1}, R_{2}, R_{3}, R_{4}, S>0$. The time derivatives of $V\left(x_{t}\right)$, along the trajectories of system (5) satisfy

$$
\begin{aligned}
& \dot{V}\left(x_{t}\right)=e^{2 \alpha t} \cdot\left[2 \alpha \cdot(x(t)-D x(t-\tau))^{T} P(x(t)-D x(t-\tau))\right] \\
& +2 e^{2 \alpha t} \cdot \sum_{i=1}^{N} \lambda_{i}(t)\left[\left\{\left[A_{0 i}+\Delta A_{0 i}(t)\right] x(t)\right\}^{T} P(x(t)-D x(t-\tau))\right] \\
& +2 e^{2 \alpha t} \cdot \sum_{i=1}^{N} \lambda_{i}(t)\left[\left\{\left[A_{1 i}+\Delta A_{1 i}(t)\right] x(t-h(t))\right\}^{T} P(x(t)-D x(t-\tau))\right] \\
& +e^{2 \alpha t} \cdot\left[x^{T}(t) Q_{1} x(t)-e^{-2 \alpha h_{m}} \cdot x^{T}\left(t-h_{m}\right) Q_{1} x\left(t-h_{m}\right)\right] \\
& +e^{2 \alpha t} \cdot\left[x^{T}(t) Q_{2} x(t)-(1-\dot{h}(t)) \cdot e^{-2 \alpha h(t)} \cdot x^{T}(t-h(t)) Q_{2} x(t-h(t))\right] \\
& +e^{2 \alpha t} \cdot\left[x^{T}(t) S x(t)-e^{-2 \alpha \tau} \cdot x^{T}(t-\tau) S x(t-\tau)\right] \\
& +e^{2 \alpha t} \cdot\left[h_{m} \cdot \dot{x}^{T}(t) R_{1} \dot{x}(t)-\int_{t-h_{m}}^{t} e^{2 \alpha(s-t)} \cdot \dot{x}^{T}(s) R_{1} \dot{x}(s) d s\right] \\
& +e^{2 \alpha t} \cdot\left[\left(h_{M}-h_{m}\right) \cdot \dot{x}^{T}(t) R_{2} \dot{x}(t)-\int_{t-h_{M}}^{t-h_{m}} e^{2 \alpha(s-t)} \cdot \dot{x}^{T}(s) R_{2} \dot{x}(s) d s\right] \\
& +e^{2 \alpha t} \cdot\left[e^{-2 \alpha h_{m}} x^{T}\left(t-h_{m}\right) R_{3} x\left(t-h_{m}\right)\right. \\
& \left.\quad-e^{-2 \alpha h_{M}} x^{T}\left(t-h_{M}\right) R_{3} x\left(t-h_{M}\right)\right]
\end{aligned}
$$$$
+e^{2 \alpha t} \cdot\left[x^{T}(t) R_{4} x(t)-e^{-2 \alpha h_{M}} x^{T}\left(t-h_{M}\right) R_{4} x\left(t-h_{M}\right)\right]
$$ 
By some simple derivatives, we have

$$
-\int_{t-h_{m}}^{t} e^{2 \alpha(s-t)} \cdot \dot{x}^{T}(s) R_{1} \dot{x}(s) d s \leq-e^{-2 \alpha h_{m}} \cdot \int_{t-h_{m}}^{t} \dot{x}^{T}(s) R_{1} \dot{x}(s) d s,
$$

and

$$
\begin{aligned}
& -\int_{t-h_{M}}^{t-h_{m}} e^{2 \alpha(s-t)} \cdot \dot{x}^{T}(s) R_{2} \dot{x}(s) d s \\
& =-e^{-2 \alpha h_{M}} \cdot\left(\int_{t-h_{M}}^{t-h(t)} \dot{x}^{T}(s) R_{2} \dot{x}(s) d s+\int_{t-h(t)}^{t-h_{m}} \dot{x}^{T}(s) R_{2} \dot{x}(s) d s\right) .
\end{aligned}
$$

Define

$$
\begin{aligned}
\hat{X}^{T}=\left[\begin{array}{llll}
x^{T}(t) & x^{T}(t-\tau) & x^{T}(t-h(t)) & x^{T}\left(t-h_{m}\right) \\
\dot{x}^{T}(t) & \dot{x}^{T}(t-\tau) & x^{T}\left(t-h_{M}\right)
\end{array}\right] .
\end{aligned}
$$

By Leibniz-Newton formula and LMIs (6b), we have

$$
\begin{aligned}
& \int_{t-h_{m}}^{t}\left[\begin{array}{c}
\hat{X} \\
\dot{x}(s)
\end{array}\right]^{T}\left[\begin{array}{cc}
R_{11} & R_{12} \\
* & R_{22}
\end{array}\right]\left[\begin{array}{c}
\hat{X} \\
\dot{x}(s)
\end{array}\right] d s \\
& =h_{m} \hat{X}^{T} R_{11} \hat{X}+2 \hat{X}^{T} R_{12}\left[x(t)-x\left(t-h_{m}\right)\right] \\
& +\int_{t-h_{m}}^{t} \dot{x}^{T}(s) R_{22} \dot{x}(s) d s \geq 0, \\
& \int_{t-h_{M}}^{t-h(t)}\left[\begin{array}{c}
\hat{X} \\
\dot{x}(s)
\end{array}\right]^{T}\left[\begin{array}{cc}
S_{11} & S_{12} \\
* & S_{22}
\end{array}\right]\left[\begin{array}{c}
\hat{X} \\
\dot{x}(s)
\end{array}\right] d s \\
& =\left(h_{M}-h(t)\right) \cdot \hat{X}^{T} S_{11} \hat{X}+2 \hat{X}^{T} S_{12}\left[x(t-h(t))-x\left(t-h_{M}\right)\right] \\
& +\int_{t-h_{M}}^{t-h(t)} \dot{x}^{T}(s) S_{22} \dot{x}(s) d s \geq 0 \\
& \int_{t-h(t)}^{t-h_{m}}\left[\begin{array}{c}
\hat{X} \\
\dot{x}(s)
\end{array}\right]^{T}\left[\begin{array}{cc}
T_{11} & T_{12} \\
* & T_{22}
\end{array}\right]\left[\begin{array}{c}
\hat{X} \\
\dot{x}(s)
\end{array}\right] d s \\
& =\left(h(t)-h_{m}\right) \cdot \hat{X}^{T} T_{11} \hat{X}+2 \hat{X}^{T} T_{12}\left[x\left(t-h_{m}\right)-x(t-h(t))\right] \\
& +\int_{t-h(t)}^{t-h_{m}} \dot{x}^{T}(s) T_{22} \dot{x}(s) d s \geq 0,
\end{aligned}
$$

By system (5), and $\lambda_{i}^{2}(t)=\lambda_{i}(t)$, and $\lambda_{i}(t) \lambda_{j}(t)=0, i \neq j$, we have

$$
-\sum_{i=1}^{N} \lambda_{i}(t)(\dot{x}(t)-D \dot{x}(t-\tau))^{T}\left(U_{1 i}+U_{1 i}^{T}\right)(\dot{x}(t)-D \dot{x}(t-\tau))
$$

$$
+\sum_{i=1}^{N} \lambda_{i}(t)\left[( \dot { x } ( t ) - D \dot { x } ( t - \tau ) ) ^ { T } U _ { 1 i } \left\{\left[A_{0 i}+\Delta A_{0 i}(t)\right] x(t)\right.\right.
$$

$$
\left.\left.+\left[A_{1 i}+\Delta A_{1 i}(t)\right] x(t-h(t))\right\}\right]
$$$$
+\sum_{i=1}^{N} \lambda_{i}(t)\left[\left\{\left[A_{0 i}+\Delta A_{0 i}(t)\right] x(t)\right\}^{T} U_{1 i}^{T}(\dot{x}(t)-D \dot{x}(t-\tau))\right]
$$$$
+\sum_{i=1}^{N} \lambda_{i}(t)\left[\left\{\left[A_{1 i}+\Delta A_{1 i}(t)\right] x(t-h(t))\right\}^{T} U_{1 i}^{T}(\dot{x}(t)-D \dot{x}(t-\tau))\right]
$$

$=0$,

$\sum_{i=1}^{N} \lambda_{i}(t) \cdot\left\{x^{T}(t) U_{2 i}^{T}+x^{T}(t-h(t)) U_{3 i}^{T}+\dot{x}^{T}(t) U_{4 i}^{T}+\dot{x}^{T}(t-\tau) U_{5 i}^{T}\right.$

$$
\left.+x^{T}\left(t-h_{m}\right) U_{6 i}^{T}+x^{T}\left(t-h_{M}\right) U_{7 i}^{T}\right\}
$$

$$
\begin{aligned}
\sum_{i=1}^{N} \lambda_{i}(t) \cdot\{ & \dot{x}(t)+D \dot{x}(t-\tau) \\
& \left.+\left[A_{0 i}+\Delta A_{0 i}(t)\right] x(t)+\left[A_{1 i}+\Delta A_{1 i}(t)\right] x(t-h(t))\right\}
\end{aligned}
$$$$
+\sum_{i=1}^{N} \lambda_{i}(t) \cdot\left\{-\dot{x}(t)+D \dot{x}(t-\tau)+\left[A_{0 i}+\Delta A_{0 i}(t)\right] x(t)\right.
$$$$
\left.+\left[A_{1 i}+\Delta A_{1 i}(t)\right] x(t-h(t))\right\}^{T}
$$

$$
\cdot \sum_{i=1}^{N} \lambda_{i}(t) \cdot\left\{x^{T}(t) U_{2 i}^{T}+x^{T}(t-h(t)) U_{3 i}^{T}+\dot{x}^{T}(t) U_{4 i}^{T}\right.
$$$$
\left.+\dot{x}^{T}(t-\tau) U_{5 i}^{T}+x^{T}\left(t-h_{m}\right) U_{6 i}^{T}+x^{T}\left(t-h_{M}\right) U_{7 i}^{T}\right\}^{T}
$$

$$
\begin{aligned}
& \begin{array}{l}
=\sum_{i=1}^{N} \lambda_{i}(t) \cdot\left[\left\{x^{T}(t) U_{2 i}^{T}+x^{T}(t-h(t)) U_{3 i}^{T}+\dot{x}^{T}(t) U_{4 i}^{T}\right.\right. \\
\left.\quad+\dot{x}^{T}(t-\tau) U_{5 i}^{T}+x^{T}\left(t-h_{m}\right) U_{6 i}^{T}+x^{T}\left(t-h_{M}\right) U_{7 i}^{T}\right\} \\
\cdot\left\{-\dot{x}(t)+D \dot{x}(t-\tau)+\left[A_{0 i}+\Delta A_{0 i}(t)\right] x(t)\right. \\
\left.\left.+\left[A_{1 i}+\Delta A_{1 i}(t)\right] x(t-h(t))\right\}\right]
\end{array}
\end{aligned}
$$

$+\sum_{i=1}^{N} \lambda_{i}(t) \cdot\left[\left\{-\dot{x}(t)+D \dot{x}(t-\tau)+\left[A_{0 i}+\Delta A_{0 i}(t)\right] x(t)\right.\right.$

$$
\left.+\left[A_{1 i}+\Delta A_{1 i}(t)\right] x(t-h(t))\right\}^{T}
$$




$$
\begin{aligned}
\cdot\left\{x^{T}(t) U_{2 i}^{T}+\right. & x^{T}(t-h(t)) U_{3 i}^{T}+\dot{x}^{T}(t) U_{4 i}^{T}+\dot{x}^{T}(t-\tau) U_{5 i}^{T} \\
& \left.\left.+x^{T}\left(t-h_{m}\right) U_{6 i}^{T}+x^{T}\left(t-h_{M}\right) U_{7 i}^{T}\right\}^{T}\right]=0 .
\end{aligned}
$$

By (8a)-(8h), we obtain the following result

$$
\begin{aligned}
\dot{V}\left(x_{t}\right) & \leq e^{2 \alpha t} \cdot\left\{\sum_{i=1}^{N} \lambda_{i}(t) \hat{X}^{T} \cdot \Sigma_{i} \cdot \hat{X}\right. \\
& -\int_{t-h_{m}}^{t} \dot{x}^{T}(s)\left[e^{-2 \alpha h_{m}} \cdot R_{1}-R_{22}\right] \dot{x}(s) d s \\
& -\int_{t-h_{M}}^{t-h(t)} \dot{x}^{T}(s)\left[e^{-2 \alpha h_{M}} \cdot R_{2}-S_{22}\right] \dot{x}(s) d s \\
& \left.-\int_{t-h(t)}^{t-h_{m}} \dot{x}^{T}(s)\left[e^{-2 \alpha h_{M}} \cdot R_{2}-T_{22}\right] \dot{x}(s) d s\right\},
\end{aligned}
$$

where

$\Sigma_{i}=\left[\begin{array}{ccccccc}\Sigma_{11 i} & \Sigma_{12 i} & \Sigma_{13 i} & \Sigma_{14 i} & \Sigma_{15 i} & \Sigma_{16 i} & \Sigma_{17 i} \\ * & \Sigma_{22 i} & \Sigma_{23 i} & 0 & 0 & 0 & 0 \\ * & * & \Sigma_{33 i} & \Sigma_{34 i} & \Sigma_{35 i} & \Sigma_{36 i} & \Sigma_{37 i} \\ * & * & * & \Sigma_{44 i} & \Sigma_{45 i} & \Sigma_{46 i} & 0 \\ * & * & * & * & \Sigma_{55 i} & \Sigma_{56 i} & \Sigma_{57 i} \\ * & * & * & * & * & \Sigma_{66 i} & \Sigma_{67 i} \\ * & * & * & * & * & * & \Sigma_{77 i}\end{array}\right]$

$$
+\Phi_{11}+\left[\begin{array}{c}
P M_{i}+U_{2 i}^{T} M_{i} \\
-D^{T} P M_{i} \\
U_{3 i}^{T} M_{i} \\
U_{6 i}^{T} M_{i} \\
U_{1 i} M_{i}+U_{4 i}^{T} M_{i} \\
-D^{T} U_{1 i} M_{i}+U_{5 i}^{T} M_{i} \\
U_{7 i}^{T} M_{i}
\end{array}\right] F_{i}(t)\left[\begin{array}{c}
N_{0 i}^{T} \\
0 \\
N_{1 i}^{T} \\
0 \\
0 \\
0 \\
0
\end{array}\right]^{T}
$$

$$
+\left[\begin{array}{c}
N_{0 i}^{T} \\
0 \\
N_{1 i}^{T} \\
0 \\
0 \\
0 \\
0
\end{array}\right] F_{i}^{T}(t)\left[\begin{array}{c}
P M_{i}+U_{2 i}^{T} M_{i} \\
-D^{T} P M_{i} \\
U_{3 i}^{T} M_{i} \\
U_{6 i}^{T} M_{i} \\
U_{1 i} M_{i}+U_{4 i}^{T} M_{i} \\
-D^{T} U_{1 i} M_{i}+U_{5 i}^{T} M_{i} \\
U_{7 i}^{T} M_{i}
\end{array}\right]^{T}
$$

By the Lemma 1 and schur complement of [1], conditions $\hat{\Sigma}_{i}<0, i=1,2, \cdots, N$, in (6) will imply $\Sigma_{i}<0$ in (9). From $\Sigma_{i}<0$ and LMIs (6a), we have

$$
V\left(x_{t}\right) \leq V\left(x_{0}\right), \cdots t \geq 0
$$

where

$$
\begin{aligned}
V\left(x_{0}\right)= & (x(0)-D x(-\tau))^{T} P(x(0)-D x(-\tau)) \\
& +\int_{-h_{m}}^{0} e^{2 \alpha s} \cdot x^{T}(s) Q_{1} x(s) d s \\
& +\int_{-h(0)}^{0} e^{2 \alpha s} \cdot x^{T}(s) Q_{2} x(s) d s \\
& +\int_{-\tau}^{0} e^{2 \alpha s} \cdot x^{T}(s) S x(s) d s \\
& +\int_{-h_{m}}^{0} e^{2 \alpha s} \cdot\left(s+h_{m}\right) \dot{x}^{T}(s) R_{1} \dot{x}(s) d s \\
& +\int_{-h_{M}}^{-h_{m}} e^{2 \alpha s} \cdot\left(s+h_{M}\right) \dot{x}^{T}(s) R_{2} \dot{x}(s) d s \\
& +\left(h_{M}-h_{m}\right) \cdot \int_{-h_{m}}^{0} e^{2 \alpha s} \cdot \dot{x}^{T}(s) R_{2} \dot{x}(s) d s \\
& +\int_{-h_{M}}^{-h_{m}} e^{2 \alpha s} x^{T}(s) R_{3} x(s) d s \\
& +\int_{-h_{M}}^{0} e^{2 \alpha s} x^{T}(s) R_{4} x(s) d s \\
& \leq \delta_{1} \cdot\left\|x_{0}\right\|_{s}^{2},
\end{aligned}
$$

and

$$
\begin{aligned}
\delta_{1}= & \lambda_{\max }(P) \cdot(1+\|D\|)^{2}+h_{m} \cdot \lambda_{\text {max }}\left(Q_{1}\right)+h_{M} \cdot \lambda_{\max }\left(Q_{2}\right) \\
& +\tau \cdot \lambda_{\max }(S)+h_{m}^{2} \cdot \lambda_{\max }\left(R_{1}\right) \\
& +\left(h_{M}-h_{m}\right)^{2} \cdot \lambda_{\max }\left(R_{2}\right)+h_{m} \cdot\left(h_{M}-h_{m}\right) \cdot \lambda_{\text {max }}\left(R_{2}\right) \\
& +\left(h_{M}-h_{m}\right) \cdot \lambda_{\max }\left(R_{3}\right)+h_{M} \cdot \lambda_{\max }\left(R_{4}\right) .
\end{aligned}
$$

On the other hand, we have

$$
\begin{gathered}
\lambda_{\text {min }}(P) \cdot e^{2 \alpha t} \cdot\|\wp(t)\|^{2} \leq e^{2 \alpha t} \cdot \wp^{T}(t) P \wp(t) \\
\leq V\left(x_{t}\right) \leq V\left(x_{0}\right) \leq \delta_{1} \cdot\left\|x_{0}\right\|_{s}^{2},
\end{gathered}
$$

where $\wp(t)=x(t)-D x(t-\tau)$. From (11), we can obtain the following result 


$$
\begin{aligned}
\|x(t)\|= & \|\wp(t)+D x(t-\tau)\| \leq\|D\| \cdot\|x(t-\tau)\|+\|\wp(t)\| \\
& \leq\|D\| \cdot\|x(t-\tau)\|+\delta_{2} \cdot e^{-\alpha t}, \quad t \geq 0,
\end{aligned}
$$

where $\delta_{2}=\sqrt{\delta_{1} / \lambda_{\min }(P)} \cdot\left\|x_{0}\right\|_{s}$. Since $\|D\|<1$ and $\tau>0$, we can choose a sufficiently small positive constant $\xi=\alpha<$ $-(\ln \|D\|) / \tau$ satisfying $\|D\| \cdot e^{\xi \tau}<1$. By the Razumikhin-like approach of [12], we obtain the following result

$$
\begin{gathered}
\|x(t)\| \leq\left[\sup _{-h \leq \theta \leq 0}\|x(\theta)\|+\frac{\delta_{2}}{1-\|D\| e^{\xi h}}\right] \cdot e^{-\xi t} \\
\leq\left[\left\|x_{0}\right\|_{s}+\frac{\delta_{2}}{1-\|D\| e^{\xi h}}\right] \cdot e^{-\xi t}, t \geq 0 .
\end{gathered}
$$

This completes the proof.

If $D=0$, Theorem 1 can be rewritten in the following result with $S=U_{5 i}=0$.

Corollary 1. System (1) with $D=0$ and (A1), $h_{D}<1$ (resp., $h_{D} \geq 1$ or unknown) is globally exponentially stable with convergence rate $\alpha>0$, if there exist some $n \times n$ matrices $P, Q_{1}, Q_{2}, R_{1}, R_{2}, R_{3}, R_{4}, R_{22}, S_{22}, T_{22}>0, R_{11}, S_{11}, T_{11}>0 \in$ $\Re^{5 n \times 5 n}$ (resp., $Q_{2}=0$ ), some matrices $U_{1 i}, U_{2 i}, U_{3 i}, U_{4 i}, U_{6 i}$, $U_{7 i} \in \Re^{n \times n}, R_{12}, S_{12}, T_{12} \in \Re^{5 n \times n}$, and some positive constants $\varepsilon_{i}, i=1,2, \cdots, N$, such that the following LMI conditions hold for all $i=1,2, \cdots, N$

$$
\begin{aligned}
& e^{-2 \alpha h_{m}} \cdot R_{1}-R_{22}>0, e^{-2 \alpha h_{M}} \cdot R_{2}-S_{22}>0, e^{-2 \alpha h_{M}} \cdot R_{2}-T_{22}>0, \\
& {\left[\begin{array}{cc}
R_{11} & R_{12} \\
* & R_{22}
\end{array}\right]>0,\left[\begin{array}{cc}
S_{11} & S_{12} \\
* & S_{22}
\end{array}\right]>0,\left[\begin{array}{cc}
T_{11} & T_{12} \\
* & T_{22}
\end{array}\right]>0,} \\
& \Xi_{i}=\left[\begin{array}{ccccccc}
\Xi_{11 i} & \Xi_{12 i} & \Xi_{13 i} & \Xi_{14 i} & \Xi_{15 i} & \Xi_{16 i} & \Xi_{17 i} \\
* & \Xi_{22 i} & \Xi_{23 i} & \Xi_{24 i} & \Xi_{25 i} & \Xi_{26 i} & \Xi_{27 i} \\
* & * & \Xi_{33 i} & \Xi_{34 i} & 0 & \Xi_{36 i} & 0 \\
* & * & * & \Xi_{44 i} & \Xi_{45 i} & \Xi_{46 i} & 0 \\
* & * & * & * & \Xi_{55 i} & \Xi_{56 i} & 0 \\
* & * & * & * & * & \Xi_{66 i} & 0 \\
* & * & * & * & * & * & \Xi_{77 i}
\end{array}\right] \\
& +\left[\begin{array}{ccc}
\tilde{\Phi}_{11} & 0 & 0 \\
0 & 0 & 0 \\
0 & 0 & 0
\end{array}\right]<0,
\end{aligned}
$$

where

$$
\begin{aligned}
& \Xi_{11 i}=2 \alpha \cdot P+P A_{0 i}+A_{0 i}^{T} P+Q_{1}+Q_{2}+U_{2 i}^{T} A_{0 i}+A_{0 i}^{T} U_{2 i}+R_{4}, \\
& \Xi_{12 i}=P A_{1 i}+U_{2 i}^{T} A_{1 i}+A_{0 i}^{T} U_{3 i}, \Xi_{13 i}=A_{0 i}^{T} U_{6 i}, \\
& \Xi_{14 i}=A_{0 i}^{T} U_{1 i}^{T}-U_{2 i}^{T}+A_{0 i}^{T} U_{4 i}, \Xi_{15 i}=A_{0 i}^{T} U_{7 i} \\
& \Xi_{16 i}=P M_{i}+U_{2 i}^{T} M_{i}, \Xi_{17 i}=\varepsilon_{i} \cdot N_{0 i}^{T}, \\
& \Xi_{22 i}=-e^{-2 \alpha h_{M}} \cdot\left(1-h_{D}\right) \cdot Q_{2}+U_{3 i}^{T} A_{1 i}+A_{1 i}^{T} U_{3 i}, \Xi_{23 i}=A_{1 i}^{T} U_{6 i}, \\
& \Xi_{24 i}=A_{1 i}^{T} U_{1 i}^{T}-U_{3 i}^{T}+A_{1 i}^{T} U_{4 i}, \Xi_{25 i}=A_{1 i}^{T} U_{7 i}, \\
& \Xi_{26 i}=U_{3 i}^{T} M_{i}, \Xi_{27 i}=\varepsilon_{i} \cdot N_{1 i}^{T}, \Xi_{33 i}=-e^{-2 \alpha h_{m}} \cdot Q_{1}+e^{-2 \alpha h_{m}} R_{3}, \\
& \Xi_{34 i}=-U_{6 i}^{T}, \Xi_{36 i}=U_{6 i}^{T} M_{i}, \\
& \Xi_{44 i}=h_{m} \cdot R_{1}+\left(h_{M}-h_{m}\right) \cdot R_{2}-U_{1 i}-U_{1 i}^{T}-U_{4 i}-U_{4 i}^{T}, \\
& \Xi_{45 i}=-U_{7 i}, \Xi_{46 i}=U_{1 i} M_{i}+U_{4 i}^{T} M_{i}, \Xi_{55 i}=-e^{-2 \alpha h_{M}}\left(R_{3}+R_{4}\right), \\
& \Xi_{56 i}=U_{7 i}^{T} M_{i}, \Xi_{66 i}=\Xi_{77 i}=-\varepsilon_{i} \cdot I, \\
& \tilde{\Phi}_{11}=h_{m} \cdot R_{11}+R_{12} \tilde{\Lambda}_{1}+\tilde{\Lambda}_{1}^{T} R_{12}^{T}+\left(h_{M}-h_{m}\right) \cdot\left(T_{11}+S_{11}\right) \\
& +S_{12} \tilde{\Lambda}_{2}+\tilde{\Lambda}_{2}^{T} S_{12}^{T}+T_{12} \tilde{\Lambda}_{3}+\tilde{\Lambda}_{3}^{T} T_{12}^{T} \\
& \tilde{\Lambda}_{1}=\left[\begin{array}{lllll}
I & 0 & -I & 0 & 0
\end{array}\right]_{n \times 5 n}, \quad \tilde{\Lambda}_{2}=\left[\begin{array}{llllll}
0 & I & 0 & 0 & -I
\end{array}\right]_{n \times 5 n}, \\
& \tilde{\Lambda}_{3}=\left[\begin{array}{lllll}
0 & -I & I & 0 & 0
\end{array}\right]_{n \times 5 n} .
\end{aligned}
$$

In the next, we will consider the delay-dependent condition for stability of system (1) with (A2).

Theorem 2. System (1) with (A2) and $h_{D}<1$ (resp., $h_{D} \geq 1$ or unknown) is globally exponentially stable with convergence rate $0<\alpha<-(\ln \|D\|) / \tau$, if $\|D\|<1$ and there exist some $n \times$ $n$ matrices $P, Q_{1}, Q_{2}, R_{1}, R_{2}, R_{3}, R_{4}, S R_{22}, S_{22}, T_{22}>0, R_{11}, S_{11}$, $T_{11}>0 \in \Re^{7 n \times 7 n}$ (resp., $Q_{2}=0$ ), some matrices $U_{1 i}, U_{2 i}, U_{3 i}$, $U_{4 i}, U_{5 i}, U_{6 i}, U_{7 i} \in \Re^{n \times n}, R_{12}, S_{12}, T_{12} \in \Re^{7 n \times n}$, and some positive constants $\mu_{0 i}, \quad \mu_{1 i}, \quad i=1,2, \cdots, N$, such that the following LMI conditions hold for all $i=1,2, \cdots, N$

$$
\begin{gathered}
e^{-2 \alpha h_{m}} \cdot R_{1}-R_{22}>0, e^{-2 \alpha h_{M}} \cdot R_{2}-S_{22}>0, e^{-2 \alpha h_{M}} \cdot R_{2}-T_{22}>0, \\
{\left[\begin{array}{cc}
R_{11} & R_{12} \\
* & R_{22}
\end{array}\right]>0,\left[\begin{array}{cc}
S_{11} & S_{12} \\
* & S_{22}
\end{array}\right]>0,\left[\begin{array}{cc}
T_{11} & T_{12} \\
* & T_{22}
\end{array}\right]>0,}
\end{gathered}
$$




$$
\begin{aligned}
\Psi_{i}= & {\left[\begin{array}{ccccccccc}
\Psi_{11 i} & \Psi_{12 i} & \Psi_{13 i} & \Psi_{14 i} & \Psi_{15 i} & \Psi_{16 i} & \Psi_{17 i} & \Psi_{18 i} & \Psi_{19 i} \\
* & \Psi_{22 i} & \Psi_{23 i} & 0 & 0 & 0 & 0 & \Psi_{28 i} & \Psi_{29 i} \\
* & * & \Psi_{33 i} & \Psi_{34 i} & \Psi_{35 i} & \Psi_{36 i} & \Psi_{37 i} & \Psi_{38 i} & \Psi_{39 i} \\
* & * & * & \Psi_{44 i} & \Psi_{45 i} & \Psi_{46 i} & 0 & \Psi_{48 i} & \Psi_{49 i} \\
* & * & * & * & \Psi_{55 i} & \Psi_{56 i} & \Psi_{57 i} & \Psi_{58 i} & \Psi_{59 i} \\
* & * & * & * & * & \Psi_{66 i} & \Psi_{67 i} & \Psi_{68 i} & \Psi_{69 i} \\
* & * & * & * & * & * & \Psi_{77 i} & \Psi_{78 i} & \Psi_{79 i} \\
* & * & * & * & * & * & * & \Psi_{88 i} & 0 \\
* & * & * & * & * & * & * & * & \Psi_{99 i}
\end{array}\right] } \\
& +\left[\begin{array}{ccc}
\Phi_{11} & 0 & 0 \\
0 & 0 & 0 \\
0 & 0 & 0
\end{array}\right]<0,
\end{aligned}
$$

where

$$
\begin{aligned}
\Psi_{11 i}= & 2 \alpha \cdot P+P A_{0 i}+A_{0 i}^{T} P+Q_{1}+Q_{2} \\
& +U_{2 i}^{T} A_{0 i}+A_{0 i}^{T} U_{2 i}+S+\mu_{0 i} \cdot \sigma_{0 i}^{2} \cdot I+R_{4}, \\
\Psi_{12 i}= & -2 \alpha \cdot P D-A_{0 i}^{T} P D, \\
\Psi_{13 i}= & P A_{1 i}+U_{2 i}^{T} A_{1 i}+A_{0 i}^{T} U_{3 i}, \Psi_{14 i}=A_{0 i}^{T} U_{6 i}, \\
\Psi_{15 i}= & A_{0 i}^{T} U_{1 i}^{T}-U_{2 i}^{T}+A_{0 i}^{T} U_{4 i}, \\
\Psi_{16 i}= & -A_{0 i}^{T} U_{1 i}^{T} D+U_{2 i}^{T} D+A_{0 i}^{T} U_{5 i}, \Psi_{17 i}=A_{0 i}^{T} U_{7 i}, \\
\Psi_{18 i}= & \Psi_{19 i}=P+U_{2 i}^{T}, \Psi_{22 i}=2 \alpha \cdot D^{T} P D-e^{-2 \alpha \tau} \cdot S, \\
\Psi_{23 i}= & -D^{T} P A_{1 i}, \Psi_{28 i}=\Psi_{29 i}=-D^{T} P, \\
\Psi_{33 i}= & -e^{-2 \alpha h_{M}} \cdot\left(1-h_{D}\right) \cdot Q_{2}+U_{3 i}^{T} A_{1 i}+A_{1 i}^{T} U_{3 i}+\mu_{1 i} \cdot \sigma_{1 i}^{2} \cdot I, \\
\Psi_{34 i}= & A_{1 i}^{T} U_{6 i}, \Psi_{35 i}=A_{1 i}^{T} U_{1 i}^{T}-U_{3 i}^{T}+A_{1 i}^{T} U_{4 i}, \\
\Psi_{36 i}= & -A_{1 i}^{T} U_{1 i}^{T} D+U_{3 i}^{T} D+A_{1 i}^{T} U_{5 i}, \Psi_{37 i}=A_{1 i}^{T} U_{7 i}, \\
\Psi_{38 i}= & \Psi_{39 i}=U_{3 i}^{T}, \Psi_{44 i}=-e^{-2 \alpha h_{m}} \cdot Q_{1}+e^{-2 \alpha h_{m}} R_{3}, \Psi_{45 i}=-U_{6 i}^{T}, \\
\Psi_{46 i}= & U_{6 i}^{T} D, \Psi_{48 i}=\Psi_{49 i}=U_{6 i}^{T}, \\
\Psi_{55 i}= & h_{m} \cdot R_{1}+\left(h_{M}-h_{m}\right) \cdot R_{2}-U_{1 i}-U_{1 i}^{T}-U_{4 i}-U_{4 i}^{T}, \\
\Psi_{56 i}= & \left(U_{1 i}+U_{1 i}^{T}\right) D+U_{4 i}^{T} D-U_{5 i}, \Psi_{57 i}=-U_{7 i}, \\
\Psi_{58 i}= & \Psi_{59 i}=U_{1 i}+U_{4 i}^{T},
\end{aligned}
$$

$$
\begin{aligned}
\Psi_{66 i}= & -D^{T}\left(U_{1 i}+U_{1 i}^{T}\right) D+U_{5 i}^{T} D+D^{T} U_{5 i}, \Psi_{67 i}=D^{T} U_{7 i}, \\
\Psi_{68 i}= & \Psi_{69 i}=-D^{T} U_{1 i}+U_{5 i}^{T}, \Psi_{77 i}=-e^{-2 \alpha h_{M}}\left(R_{3}+R_{4}\right), \\
\Psi_{78 i}= & \Psi_{79 i}=U_{7 i}^{T}, \Psi_{88 i}=-\mu_{0 i} \cdot I, \Psi_{99 i}=-\mu_{1 i} \cdot I, \\
\Phi_{11}= & h_{m} \cdot R_{11}+R_{12} \Lambda_{1}+\Lambda_{1}^{T} R_{12}^{T}+\left(h_{M}-h_{m}\right) \cdot\left(T_{11}+S_{11}\right) \\
& +S_{12} \Lambda_{2}+\Lambda_{2}^{T} S_{12}^{T}+T_{12} \Lambda_{3}+\Lambda_{3}^{T} T_{12}^{T}, \\
\Lambda_{1}= & {\left[\begin{array}{lllllll}
I & 0 & 0 & -I & 0 & 0 & 0
\end{array}\right]_{n \times 7 n}, } \\
\Lambda_{2}= & {\left[\begin{array}{lllllll}
0 & 0 & I & 0 & 0 & 0 & -I
\end{array}\right]_{n \times 7 n}, } \\
\Lambda_{3}= & {\left[\begin{array}{lllllll}
0 & 0 & -I & I & 0 & 0 & 0
\end{array}\right]_{n \times 7 n} . }
\end{aligned}
$$

Proof. From the assumption in (A2), we have

$$
\sigma_{0 i}^{2} x^{T}(t) x(t)-x^{T}(t) \Delta A_{0 i}^{T}(t) \Delta A_{0 i}(t) x(t) \geq 0,
$$

$$
\sigma_{1 i}^{2} x^{T}(t-h(t)) x(t-h(t))
$$

$$
-x^{T}(t-h(t)) \Delta A_{1 i}^{T}(t) \Delta A_{1 i}(t) x(t-h(t)) \geq 0, i=1,2, \cdots, N .
$$

By (8a)-(8h), we can obtain the following result

$$
\begin{aligned}
& \dot{V}\left(x_{t}\right)+e^{2 \alpha t} \cdot \sum_{i=1}^{N} \lambda_{i}(t) \cdot \mu_{0 i} \cdot\left[\sigma_{0 i}^{2} x^{T}(t) x(t)\right.\left.-x^{T}(t) \Delta A_{0 i}^{T}(t) \Delta A_{0 i}(t) x(t)\right] \\
&+e^{2 \alpha t} \cdot \sum_{i=1}^{N} \lambda_{i}(t) \cdot \mu_{1 i} \cdot\left[\sigma_{1 i}^{2} x^{T}(t-h(t)) x(t-h(t))\right.\left.-x^{T}(t-h(t)) \Delta A_{1 i}^{T}(t) \Delta A_{1 i}(t) x(t-h(t))\right] \\
& \leq e^{2 \alpha t} \cdot\left\{\sum_{i=1}^{n} Z_{i}^{T} \cdot \Psi_{i} \cdot Z_{i}\right. \\
& \quad-\int_{t-h_{m}}^{t} \dot{x}^{T}(s)\left[e^{-2 \alpha h_{m}} \cdot R_{1}-R_{22}\right] \dot{x}(s) d s \\
& \quad-\int_{t-h_{M}}^{t-h(t)} \dot{x}^{T}(s)\left[e^{-2 \alpha h_{M}} \cdot R_{2}-S_{22}\right] \dot{x}(s) d s \\
&\left.\quad-\int_{t-h(t)}^{t-h_{m}} \dot{x}^{T}(s)\left[e^{-2 \alpha h_{M}} \cdot R_{2}-T_{22}\right] \dot{x}(s) d s\right\},
\end{aligned}
$$


where matrices $\Psi_{i}, i=1,2, \cdots, N$, are defined in (13), and

$$
\begin{aligned}
& Z_{i}^{T}=\left[\begin{array}{lllll}
x^{T}(t) & x^{T}(t-\tau) & x^{T}(t-h(t)) & x^{T}\left(t-h_{m}\right) & \dot{x}^{T}(t)
\end{array}\right. \\
& \left.\dot{x}^{T}(t-\tau) \quad x^{T}\left(t-h_{M}\right) \quad x^{T}(t) \Delta A_{0 i}^{T}(t) \quad x^{T}(t-h(t)) \Delta A_{1 i}^{T}(t)\right]
\end{aligned}
$$

From conditions in (13)-(15), we can achieve that the condition (10) is satisfied. By the same derivation of Theorem 1, we can complete this proof.

If $D=0$, Theorem 2 can be rewritten in the following result with $S=U_{5 i}=0$.

Corollary 2. System (1) with $D=0$ and (A2), $h_{D}<1$ (resp., $h_{D} \geq 1$ or unknown) is globally exponentially stable with convergence rate $\alpha>0$, if there exist some $n \times n$ matrices $P, Q_{1}, Q_{2}, R_{1}, R_{2}, R_{3}, R_{4}, R_{22}, S_{22}, T_{22}>0, R_{11}, S_{11}, T_{11}>0 \in$ $\Re^{5 n \times 5 n}$, (resp., $Q_{2}=0$ ), some matrices $U_{1 i}, U_{2 i}, U_{3 i}, U_{4 i}, U_{6 i}$, $U_{7 i} \in \Re^{n \times n}, R_{12}, S_{12}, T_{12} \in \mathfrak{R}^{5 n \times n}$, and some positive constants $\mu_{0 i}, \mu_{1 i}, i=1,2, \cdots, N$, such that the following LMI conditions hold for all $i=1,2, \cdots, N$

$$
\begin{aligned}
& e^{-2 \alpha h_{m}} \cdot R_{1}-R_{22}>0, e^{-2 \alpha h_{M}} \cdot R_{2}-S_{22}>0, e^{-2 \alpha h_{M}} \cdot R_{2}-T_{22}>0 \\
& {\left[\begin{array}{cc}
R_{11} & R_{12} \\
* & R_{22}
\end{array}\right]>0,\left[\begin{array}{cc}
S_{11} & S_{12} \\
* & S_{22}
\end{array}\right]>0,\left[\begin{array}{cc}
T_{11} & T_{12} \\
* & T_{22}
\end{array}\right]>0 } \\
& \hat{\Psi}_{i}= {\left[\begin{array}{ccccccc}
\hat{\Psi}_{11 i} & \hat{\Psi}_{12 i} & \hat{\Psi}_{13 i} & \hat{\Psi}_{14 i} & \hat{\Psi}_{15 i} & \hat{\Psi}_{16 i} & \hat{\Psi}_{17 i} \\
* & \hat{\Psi}_{22 i} & \hat{\Psi}_{23 i} & \hat{\Psi}_{24 i} & \hat{\Psi}_{25 i} & \hat{\Psi}_{26 i} & \hat{\Psi}_{27 i} \\
* & * & \hat{\Psi}_{33 i}^{\prime} & \hat{\Psi}_{34 i} & 0 & \hat{\Psi}_{36 i} & \hat{\Psi}_{37 i} \\
* & * & * & \hat{\Psi}_{44 i} & \hat{\Psi}_{45 i} & \hat{\Psi}_{46 i} & \hat{\Psi}_{47 i} \\
* & * & * & * & \hat{\Psi}_{55 i}^{\prime} & \hat{\Psi}_{56 i} & \hat{\Psi}_{57 i} \\
* & * & * & * & * & \hat{\Psi}_{66 i} & 0 \\
* & * & * & * & * & * & \hat{\Psi}_{77 i}
\end{array}\right] } \\
&+ {\left[\begin{array}{ccc}
\tilde{\Phi}_{11} & 0 & 0 \\
0 & 0 & 0 \\
0 & 0 & 0
\end{array}\right]<0, } \\
&
\end{aligned}
$$

where

$$
\begin{aligned}
\hat{\Psi}_{11 i}= & 2 \alpha \cdot P+P A_{0 i}+A_{0 i}^{T} P+Q_{1}+Q_{2} \\
& +U_{2 i}^{T} A_{0 i}+A_{0 i}^{T} U_{2 i}+\mu_{0 i} \cdot \sigma_{0 i}^{2} \cdot I+R_{4}, \\
\hat{\Psi}_{12 i}= & P A_{1 i}+U_{2 i}^{T} A_{1 i}+A_{0 i}^{T} U_{3 i}, \\
\hat{\Psi}_{13 i}= & A_{0 i}^{T} U_{6 i}, \hat{\Psi}_{14 i}=A_{0 i}^{T} U_{1 i}^{T}-U_{2 i}^{T}+A_{0 i}^{T} U_{4 i}, \hat{\Psi}_{15 i}=A_{0 i}^{T} U_{7 i},
\end{aligned}
$$

$\hat{\Psi}_{16 i}=\hat{\Psi}_{17 i}=P+U_{2 i}^{T}$,

$\hat{\Psi}_{22 i}=-e^{-2 \alpha h_{M}} \cdot\left(1-h_{D}\right) \cdot Q_{2}+U_{3 i}^{T} A_{1 i}+A_{1 i}^{T} U_{3 i}+\mu_{1 i} \cdot \sigma_{1 i}^{2} \cdot I$,

$\hat{\Psi}_{23 i}=A_{1 i}^{T} U_{6 i}, \hat{\Psi}_{24 i}=A_{1 i}^{T} U_{1 i}^{T}-U_{3 i}^{T}+A_{1 i}^{T} U_{4 i}$,

$\hat{\Psi}_{25 i}=A_{1 i}^{T} U_{7 i}, \hat{\Psi}_{26 i}=\hat{\Psi}_{27 i}=U_{3 i}^{T}$,

$\hat{\Psi}_{33 i}=-e^{-2 \alpha h_{m}} \cdot Q_{1}+e^{-2 \alpha h_{m}} R_{3}, \hat{\Psi}_{34 i}=-U_{6 i}^{T}, \hat{\Psi}_{36 i}=\hat{\Psi}_{37 i}=U_{6 i}^{T}$,

$\hat{\Psi}_{44 i}=h_{m} \cdot R_{1}+\left(h_{M}-h_{m}\right) \cdot R_{2}-U_{1 i}-U_{1 i}^{T}-U_{4 i}-U_{4 i}^{T}$,

$\hat{\Psi}_{45 i}=-U_{7 i}, \hat{\Psi}_{46 i}=\hat{\Psi}_{47 i}=U_{1 i}+U_{4 i}^{T}$,

$\hat{\Psi}_{55 i}=-e^{-2 \alpha h_{M}}\left(R_{3}+R_{4}\right), \hat{\Psi}_{56 i}=\hat{\Psi}_{57 i}=U_{7 i}^{T}$,

$\hat{\Psi}_{66 i}=-\mu_{0 i} \cdot I, \hat{\Psi}_{77 i}=-\mu_{1 i} \cdot I$,

$\tilde{\Phi}_{11}=h_{m} \cdot R_{11}+R_{12} \tilde{\Lambda}_{1}+\tilde{\Lambda}_{1}^{T} R_{12}^{T}+\left(h_{M}-h_{m}\right) \cdot\left(T_{11}+S_{11}\right)$

$+S_{12} \tilde{\Lambda}_{2}+\tilde{\Lambda}_{2}^{T} S_{12}^{T}+T_{12} \tilde{\Lambda}_{3}+\tilde{\Lambda}_{3}^{T} T_{12}^{T}$

$\tilde{\Lambda}_{1}=\left[\begin{array}{lllll}I & 0 & -I & 0 & 0\end{array}\right]_{n \times 5 n}, \quad \tilde{\Lambda}_{2}=\left[\begin{array}{lllll}0 & I & 0 & 0 & -I\end{array}\right]_{n \times 5 n}$,

$\tilde{\Lambda}_{3}=\left[\begin{array}{lllll}0 & -I & I & 0 & 0\end{array}\right]_{n \times 5 n}$.

If $D=0, \Delta A_{0 i}(t)=0$, and $\Delta A_{1 i}(t)=0$, Corollaries 1 and 2 can be rewritten in the following result.

Corollary 3. System (1) with $D=0, \Delta A_{0 i}(t)=0, \Delta A_{1 i}(t)=0$, and $h_{D}<1$ (resp., $h_{D} \geq 1$ or unknown) is globally exponentially stable with convergence rate $\alpha>0$, if there exist some $n \times n$ matrices $P, Q_{1}, Q_{2}, R_{1}, R_{2}, R_{3}, R_{4}, R_{22}, S_{22}, T_{22}>0, R_{11}, S_{11}, T_{11}$ $>0 \in \Re^{5 n \times 5 n}$, (resp., $Q_{2}=0$ ), some matrices $U_{1 i}, U_{2 i}, U_{3 i}, U_{4 i}$, $U_{6 i}, U_{7 i} \in \Re^{n \times n}, R_{12}, S_{12}, T_{12} \in \mathfrak{R}^{5 n \times n}$, such that the following LMI conditions hold for all $i=1,2, \cdots, N$

$$
e^{-2 \alpha h_{m}} \cdot R_{1}-R_{22}>0, e^{-2 \alpha h_{M}} \cdot R_{2}-S_{22}>0, e^{-2 \alpha h_{M}} \cdot R_{2}-T_{22}>0,
$$

$$
\begin{aligned}
& {\left[\begin{array}{cc}
R_{11} & R_{12} \\
* & R_{22}
\end{array}\right]>0,\left[\begin{array}{cc}
S_{11} & S_{12} \\
* & S_{22}
\end{array}\right]>0,\left[\begin{array}{cc}
T_{11} & T_{12} \\
* & T_{22}
\end{array}\right]>0,} \\
& \hat{\Xi}_{i}=\left[\begin{array}{ccccc}
\Xi_{11 i} & \Xi_{12 i} & \Xi_{13 i} & \Xi_{14 i} & \Xi_{15 i} \\
* & \Xi_{22 i} & \Xi_{23 i} & \Xi_{24 i} & \Xi_{25 i} \\
* & * & \Xi_{33 i} & \Xi_{34 i} & 0 \\
* & * & * & \Xi_{44 i} & \Xi_{45 i} \\
* & * & * & * & \Xi_{55 i}
\end{array}\right]+\tilde{\Phi}_{11}<0,
\end{aligned}
$$




$$
\begin{aligned}
\tilde{\Phi}_{11}= & h_{m} \cdot R_{11}+R_{12} \tilde{\Lambda}_{1}+\tilde{\Lambda}_{1}^{T} R_{12}^{T}+\left(h_{M}-h_{m}\right) \cdot\left(T_{11}+S_{11}\right) \\
& +S_{12} \tilde{\Lambda}_{2}+\tilde{\Lambda}_{2}^{T} S_{12}^{T}+T_{12} \tilde{\Lambda}_{3}+\tilde{\Lambda}_{3}^{T} T_{12}^{T}, \\
\tilde{\Lambda}_{1}= & {\left[\begin{array}{lllll}
I & 0 & -I & 0 & 0
\end{array}\right]_{n \times 5 n}, \tilde{\Lambda}_{2}=\left[\begin{array}{lllll}
0 & I & 0 & 0 & -I
\end{array}\right]_{n \times 5 n}, } \\
\tilde{\Lambda}_{3}= & {\left[\begin{array}{lllll}
0 & -I & I & 0 & 0
\end{array}\right]_{n \times 5 n}, }
\end{aligned}
$$

where $\Xi_{k l i}, k, l=1,2, \ldots, 5, i=1,2, \ldots, N$, are defined in (12).

Remark 1. In [7] and [11], the slow variation condition $\dot{h}(t) \leq h_{D}<1$ is constrained in their considered systems. The hard constraint $h_{D}<1$ is not imposed on our results.

Remark 2. By setting $\alpha=0$ in Theorems 1-2 and Corollaries 1-3, the global asymptotic stability for system (1) can be guaranteed. By setting $\alpha=0, R_{1}=0$, and $R_{2}=0, R_{11}=T_{11}=S_{11}=0$ in Theorems 1-2 and Corollaries 1-3, we can obtain the delay-independent stability result for system (1).

\section{NUMERICAL EXAMPLES}

Example 1. Consider the system (1) with $D=0, \Delta A_{0 i}(t)=0$, and $\Delta A_{1 i}(t)=0$ and the following parameters: (Example 1 of [9])

$$
\begin{gathered}
N=3, A_{01}=\left[\begin{array}{cc}
-2 & 0 \\
0 & -1
\end{array}\right], A_{11}=\left[\begin{array}{cc}
-1 & -1 \\
0 & -0.5
\end{array}\right], A_{02}=\left[\begin{array}{cc}
-1.5 & 1 \\
0 & -1
\end{array}\right], \\
A_{12}=\left[\begin{array}{cc}
0.6 & -1 \\
0 & -0.4
\end{array}\right], A_{03}=\left[\begin{array}{cc}
-0.5 & 0 \\
0 & -3
\end{array}\right], A_{13}=\left[\begin{array}{cc}
-1 & 1 \\
0 & 1
\end{array}\right] .
\end{gathered}
$$

By Corollary 3, LMI (17) with (18) for $\alpha=0.1, h_{D}<0.2$, $h_{m}<0.1, h_{M}<1.1$ has a feasible solution. This implies that the system (1) with $D=1, \Delta A_{0 i}(t)=0, \Delta A_{1 i}(t)=0,0.1 \leq h(t) \leq 1.1$, $\dot{h}(t) \leq 0.2$, and (18) is globally exponentially stable with convergence rate $\alpha=0.1$. Some comparisons of the obtained results for switched system (1) with (18) are made in Table 1. The results of this paper provide a larger allowable upper bound for time delay to guarantee the global asymptotic stability of system (1) with (18).

Example 2. Consider the system (1) with $D=0, \Delta A_{0 i}(t)=0$,

\begin{tabular}{|c|c|c|}
\hline $\begin{array}{r}\text { Some results of delay } \\
\qquad(\alpha=0)\end{array}$ & $\begin{array}{l}\text { hat guarantee globa } \\
\text { f the system (1) wi }\end{array}$ & $\begin{array}{l}\text { symptotic stability } \\
\text { (18) }\end{array}$ \\
\hline Results & [9] & Our result \\
\hline$h_{D}=0$ (Constant delay) & \multirow{4}{*}{ No results } & $0 \leq h \leq 1.5004$ \\
\hline$h_{D}=0.4$ & & $0 \leq h(t) \leq 1.277$ \\
\hline$h_{D}=0.8$ & & $0 \leq h(t) \leq 1.1235$ \\
\hline$h_{D}=0.9$ & & $0 \leq h(t) \leq 1.1209$ \\
\hline$h_{D} \geq 1$ or unknown & \multirow{4}{*}{$0 \leq h(t) \leq 0.9339$} & $0 \leq h(t) \leq 1.12$ \\
\hline$h_{D} \geq 1$ or unknown & & $0.3 \leq h(t) \leq 1.17$ \\
\hline$h_{D} \geq 1$ or unknown & & $0.6 \leq h(t) \leq 1.21$ \\
\hline$h_{D} \geq 1$ or unknown & & $0.9 \leq h(t) \leq 1.28$ \\
\hline
\end{tabular}
and $\Delta A_{1 i}(t)=0$ and the following parameters: (Example 1 of [8])

\begin{tabular}{|c|c|c|}
\hline \multicolumn{3}{|c|}{$\begin{array}{l}\text { Some upper bounds of delay that guarantee the global exponential } \\
\text { stability of the system (1) with (19) }\end{array}$} \\
\hline Results & $\begin{array}{l}\text { convergence rate } \alpha \\
\quad \text { and } h_{D}\end{array}$ & Bounds of delay $h_{m}$ and $h_{M}$ \\
\hline [8] & $\alpha=0.3, h_{D}=0.1$ & $h_{m}=0, h_{M}=0.4$ \\
\hline \multirow{5}{*}{ Our results } & $\alpha=0.3, h_{D}=0.1$ & $h_{m}=0, h_{M}=0.497$ \\
\hline & \multirow{4}{*}{$\begin{array}{c}\alpha=0.3, \\
\left(h_{D} \geq 1 \text { or unknown }\right)\end{array}$} & $h_{m}=0, h_{M}=0.475$ \\
\hline & & $h_{m}=0.3, h_{M}=0.511$ \\
\hline & & $h_{m}=0.4, h_{M}=0.519$ \\
\hline & & $h_{m}=0.5, h_{M}=0.525$ \\
\hline \multicolumn{3}{|c|}{$\begin{array}{l}\text { Some upper bounds of delay that guarantee the global asymptotic } \\
\text { stability of the system (1) with (19) }\end{array}$} \\
\hline [8] & $\alpha=0, h_{D}=0.1$ & $h_{m}=0, h_{M}=1$ \\
\hline \multirow{5}{*}{ Our results } & $\alpha=0, h_{D}=0.1$ & $h_{m}=0, h_{M}=3.585$ \\
\hline & \multirow{4}{*}{$\begin{array}{c}\alpha=0 \\
\left(h_{D} \geq 1 \text { or unknown }\right)\end{array}$} & $h_{m}=0, h_{M}=0.895$ \\
\hline & & $h_{m}=0.4, h_{M}=1.08$ \\
\hline & & $h_{m}=0.8, h_{M}=1.35$ \\
\hline & & $h_{m}=1, h_{M}=1.501$ \\
\hline
\end{tabular}

$$
\begin{gathered}
N=2, A_{01}=A_{02}=\left[\begin{array}{cc}
-1 & 0 \\
0 & -1
\end{array}\right], A_{11}=\left[\begin{array}{cc}
0 & 0.5 \\
-1 & 0
\end{array}\right], \\
A_{12}=\left[\begin{array}{cc}
0 & 1 \\
-0.5 & 0
\end{array}\right] .
\end{gathered}
$$

Table 1. Comparing some previous results with this paper.

Table 2. Comparing some previous results with this paper.

Some comparisons of the obtained results for switched system (1) with (19) are made in Table 2. The results of this paper provide a larger allowable upper bound for time delay to guarantee the global asymptotic and exponential stability of system (1) with (19).

\section{CONCLUSIONS}

In this paper, global exponential stability for uncertain switched neutral systems with interval time-varying state delay and arbitrary switching signal has been considered. Structured and unstructured perturbations of systems have been investigated. LMI and Razumikhin-like approaches have been used to improve our results. The obtained results are less conservative than previous ones via the numerical simulation. 


\section{ACKNOWLEDGMENTS}

The research reported here was supported by the National Science Council of Taiwan, R.O.C. under grant no. NSC 962221-E-022-011-MY2.

\section{REFERENCES}

1. Boyd, S., Ghaoui, L. E., Feron, E., and Balakrishnan V., Linear Matrix Inequalities in System and Control Theory, Society for Industrial and Applied Mathematics (SIAM), Philadelphia, USA (1994).

2. Gu, K., Kharitonov, V. L., and Chen, J., Stability of Time-Delay Systems, Birkhauser, Boston, Massachusetts, USA (2003).

3. Hale, J. K. and Verduyn Lunel, S. M., Introduction to Functional Differential Equations, Springer-Verlag, New York, USA (1993).

4. Kim, S., Campbell, S. A., and Liu, X., "Stability of a class of linear switching systems with time delay," IEEE Transactions on Circuits Systems, Vol. 53, pp. 384-393 (2006).

5. Kolmanovskii, V. B. and Myshkis, A., Applied Theory of Functional Differential Equations, Kluwer Academic Publishers, Netherlands (1992).

6. Liu, D., Liu, X., and Zhong, S., "Delay-dependent robust stability and control synthesis for uncertain switched neutral systems with mixed de- lays," Applied Mathematics and Computation, Vol. 202, pp. $828-839$ (2008).

7. Liu, J., Liu, X., and Xie, W. C., "Delay-dependent robust control for uncertain switched systems with time-delay," Nonlinear Analysis: Hybrid Systems, Vol. 2, pp. 81-95 (2008).

8. Sun, X. M., Zhao, J., and Hill, D. J., "Stability and $L_{2}$-gain analysis for switched delay systems: A delay-dependent method," Automatica, Vol. 42, pp. 1769-1774 (2006).

9. Sun, Y. G., Wang, L., and Xie, G., "Stability of switched systems with time-varying delays: delay-dependent common Lyapunov functional approach," Proceedings of the 2006 American Control Conference, Vol. 5, pp. 1544-1549 (2006).

10. Sun, Z. and Ge, S. S., Switched Linear Systems: Control and Design, Springer-Verlag, London, UK (2005).

11. Wang, C. H., Zhang, L. X., Gao, H. J., and Wu, L. G., "Delay-dependent stability and stabilization of a class of linear switched time-varying delay systems," Proceedings of the 4th International Conference on Machine Learning and Cybernetics, pp. 917-922 (2005).

12. Yu, K. W. and Lien, C. H., "Stability criteria for uncertain neutral systems with interval time-varying delays," Chaos, Solitons and Fractals, Vol. 38, pp. 650-657 (2008)

13. Zhang, Y., Liu, X., Zhu, H., and Zhong, S., "Stability analysis and control synthesis for a class of switched neutral systems," Applied Mathematics and Computation, Vol. 190, pp. 1258-1266 (2007). 